\title{
Clinicopathologic and Magnetic Resonance Imaging Analysis of a Multifocal Orbital Lymphoid Tumor
}

\author{
Frederick A. Jakobiec ${ }^{a, c}$ Natalie Homer ${ }^{b, c}$ Fouad R. Zakkaa, c Hugh D. Curtin ${ }^{d}$ \\ Aaron Fay ${ }^{\mathrm{C}}$ \\ aDavid G. Cogan Laboratory of Ophthalmic Pathology, ${ }^{b}$ Orbital and Oculoplastics Service, ${ }^{c}$ Department of \\ Ophthalmology, and d Department of Radiology, Massachusetts Eye and Ear Infirmary and Harvard Medical School, \\ Boston, MA, USA
}

\section{Keywords}

Orbit - Lymphoid tumor - Lymphaticovenous malformation . Lymphangioma $\cdot$ Magnetic resonance imaging

\begin{abstract}
Objective: To distinguish between a multifocal orbital lymphoid tumor and a major simulator represented by a diffuse lymphaticovenous malformation. Methods: We performed a comparison of clinical and radiographic (magnetic resonance imaging [MRI]) findings of these two disparate entities and demonstrated how a misdiagnosis can be prevented. Results: Orbital lymphoid tumors develop in adults at around 60 years of age, whereas extensive lymphaticovenous malformations are generally detected in the first decade. Despite these differences, this is the first description of clinical confusion between them. MRI with gadolinium injection in the current lymphoid tumor displayed a low signal on T2weighted images, rapid and uniform enhancement, and reduced diffusion. Lymphaticovenous malformations are heterogeneous, display poor or only focal perfusion, and fail to exhibit diminished diffusion. Newer techniques such as dif-
\end{abstract}

\section{KARGER}

(c) 2017 S. Karger AG, Basel

E-Mail karger@karger.com

www.karger.com/oop fusion-weighted imaging and dynamic contrast-enhanced imaging may be able to provide additional differential diagnostic information. The final pathologic diagnosis was an extranodal marginal zone lymphoma. Conclusions: Despite the obvious distinctions between orbital lymphoid tumors and lymphaticovenous malformations, several clinical radiologic specialists misdiagnosed the present orbital lesion as a vascular lesion. A combined clinicoradiographic analysis should obviate such errors and facilitate the correct diagnosis in the future.

(c) 2017 S. Karger AG, Basel

\section{Introduction}

Despite being the most common class of primary orbital neoplasia in adults, lymphoid tumors are still frequently misdiagnosed as other conditions. The latter include idiopathic orbital inflammation or pseudotumor (the most common misdiagnosis), thyroid ophthalmopathy, orbital cellulitis, lacrimal gland epithelial tumor, dacryoadenitis, metastatic carcinoma, and optic nerve me- 
ningioma, among others [1-4]. Delays in reaching the correct diagnosis have ranged from an average of 2 months [5] to 23 months as the longest [6]. This report describes an extensive orbital lymphoid mass that in the minds of several specialists simulated a diffuse vascular malformation.

The main objective of this study is to outline the clini$\mathrm{cal}$ and radiographic features on magnetic resonance imaging (MRI) which, when properly interpreted together, should preclude the foregoing problem and promote an earlier correct diagnosis. Attention is also paid in passing to emerging radiographic techniques that may further advance diagnostic precision. Knowledge possessed by clinicians regarding these two disparate entities may not be familiar to radiologists; furthermore, the experience of many radiologists regarding the rich variety of orbital conditions may be limited. This paper is intended to synthesize information that should be valuable to both radiologists and ophthalmic clinicians. In the current case, MRI was performed and initially interpreted at an outside institution as representing a vascular malformation, while an extensive lymphoid tumor was overlooked. This situation persisted during several subsequent consultations until the patient was evaluated at our institution.

\section{Case Report}

A 38-year-old Caucasian woman presented to an ophthalmologist after experiencing several months of episodic right periorbital swelling. Her vision was 20/25 OD and 20/20 OS with intact pupillary responses and color vision. The intraocular pressures were $27 \mathrm{~mm} \mathrm{Hg} \mathrm{OD}$ and $17 \mathrm{~mm} \mathrm{Hg}$ OS. The right eye movements were full but accompanied by discomfort in far left, right, and inferior gaze. There were $5 \mathrm{~mm}$ of right eye proptosis with resistance to retropulsion with mild upper eyelid ptosis and conjunctival chemosis (Fig. 1). MRI study demonstrated a right multicentric orbital mass. The patient was referred to an orbital specialist who confirmed the results of the earlier examination. The constellation of findings was felt to be consistent with a benign vascular lesion.

The patient's symptoms progressed over the next 6 months, whereupon she sought evaluation from several additional orbital surgeons as well as a neuroophthalmologist. The prevailing diagnosis was a benign venous or lymphaticovenous malformation. The patient was seen again in consultation shortly thereafter. The examination did not reveal any spontaneous orbital pulsations, palpable orbital pulse, nor thrill.

The outside initial and only MRI study with and without gadolinium contrast administration was reviewed (Fig. 2a-f, Fig. 3a, b). Several discrete lesions were visualized in the orbit. The anterior inferior lesion molded to the globe and several other lesions appeared to follow along the orbital facial compartments. The lesions were isointense to muscle on the T1-weighted images and relatively low signal (dark) on the T2-weighted sequence. The tumor appeared to follow the optic nerve sheath, as is commonly seen in

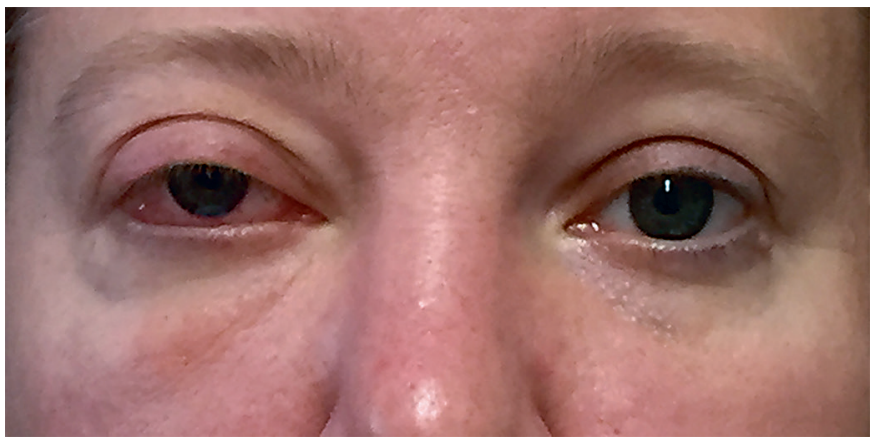

Fig. 1. Clinical presentation of the 38-year-old female patient with gradual onset of relative right proptosis with well-preserved extraocular motility and visual acuity. Note that the right superior sulcus had been filled out and that there was minimal eyelid inflammation and bland chemosis.

meningioma. There was a suggestion of a small lesion along the inferior aspect of the contralateral nerve. The lesions enhanced uniformly on imaging immediately after administration of gadolinium. Diffusion-weighted sequences showed intermediately reduced diffusion (Fig. 3a, b). This degree of detail was not included in the report of the study from the outside institution.

Transconjunctival orbitotomy revealed an infiltrative tumor in the inferior orbit with complete inferior oblique obliteration. The lesion was carefully dissected posteriorly, allowing for gross total resection. Pathologic evaluation revealed a follicular lesion composed of small to medium-sized lymphoid and plasma cells infiltrating the extraocular muscles and large peripheral nerves (Fig. 3c, d). Immunohistochemistry showed small CD10-positive follicular centers (Fig. 3e) scattered among a predominance of CD20-positive, CD10-negative, ad Bcl2-positive B lymphocytes with a kappa immunoglobulin light chain predominance (Fig. 3f), 5-10\% Ki67 reactivity, and no chromosomal aberrations. These findings were considered to be diagnostic of an extranodal marginal zone B-cell lymphoma (mucosa-associated lymphoid tissue lymphoma). The patient had a negative systemic workup and underwent localized proton beam irradiation with symptomatic improvement. There was no surgically induced morbidity and visual acuity after proton beam treatment was 20/20. Neither local recurrence nor systemic disease has emerged during 13 months of follow-up.

\section{Discussion}

Several salient clinical and radiographic distinctions exist between extensive orbital lymphoid tumors and vascular malformations that can be very helpful in differentiating them, which is unproblematic in the majority of cases. Unfortunately, ophthalmic clinicians and radiologists are not always cognizant of their full range of appearances due to the rarity of vascular malformations and some exceptional configurations - particularly multifocality - of orbital lymphoid tumors. In the current case, 

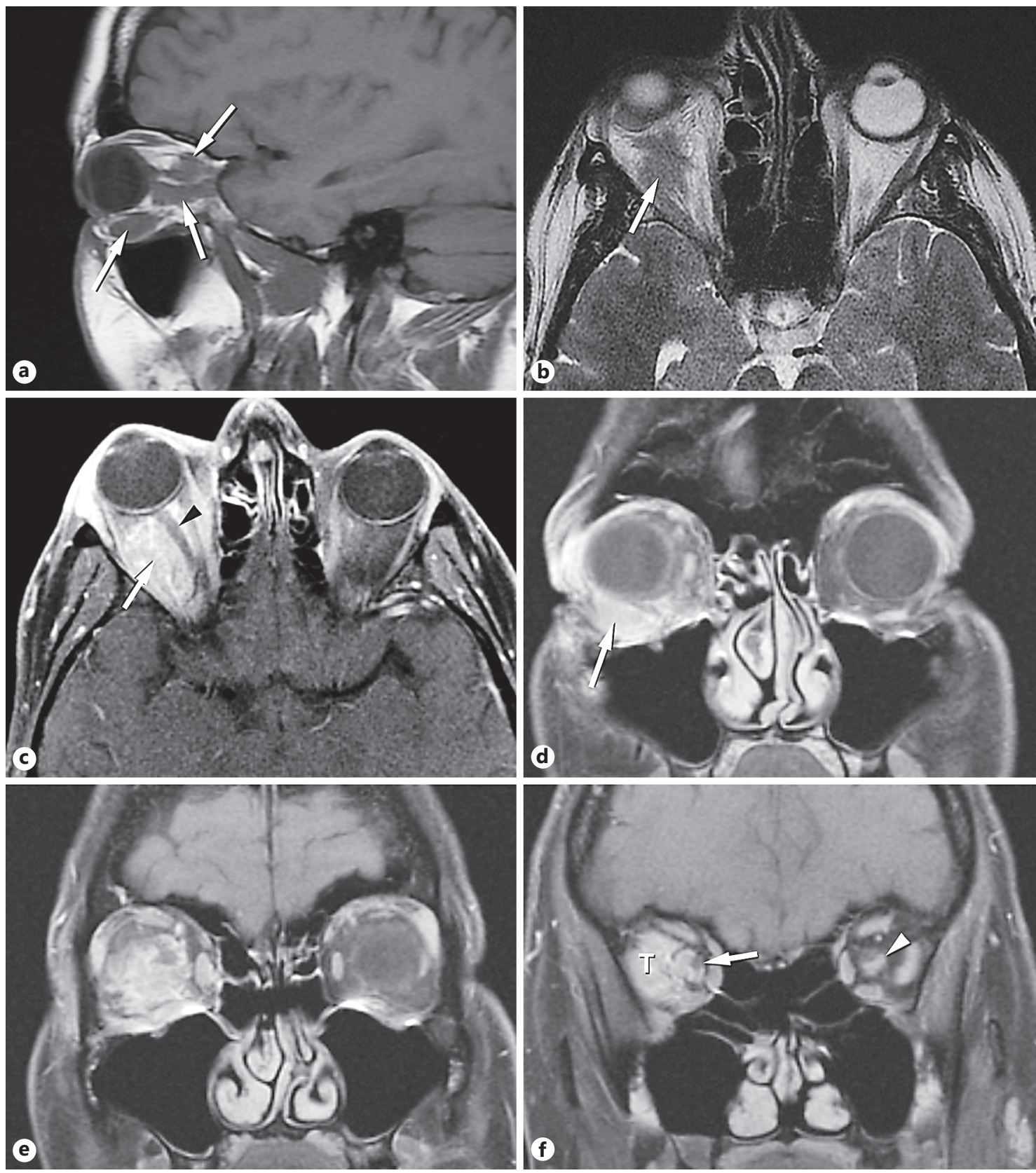

Fig. 2. Magnetic resonance imaging. a The sagittal T1-weighted image showed multiple foci of abnormal signal (arrows) contrasted against the adjacent fat. $\mathbf{b}$ The axial T2-weighted image showed iso- to low-signal intensity (arrow). c The axial post-gadolinium fat-suppressed image showed the enhancing lesion (arrow) filling much of the orbit and slightly displacing the optic nerve (arrowhead). d Coronal T1-weighted image of the anterior orbit with fat suppression after gadolinium administration. The enhancing lesion (arrow) was located inferior to the globe, displacing it superiorly. Note how the lesion molded to the wall of the globe. e Coro- nal T1-weighted image, post contrast, fat-suppressed just posterior to Figure $1 \mathrm{~d}$. The lesion enhanced and appeared to wrap partially around the optic nerve. The shape of the lesion reflected the orbital fascial layers. f Coronal T1-weighted image, post contrast, fat-suppressed image just posterior to Figure 1e. The enhancing tumor (T) filled the lateral compartment of the orbit. The tumor also extended along the optic nerve sheath. The nerve (arrow) was visualized partially surrounded by the lesion. This appearance is most commonly seen in meningioma. A small lesion (arrowhead) was present in the contralateral nerve sheath. 

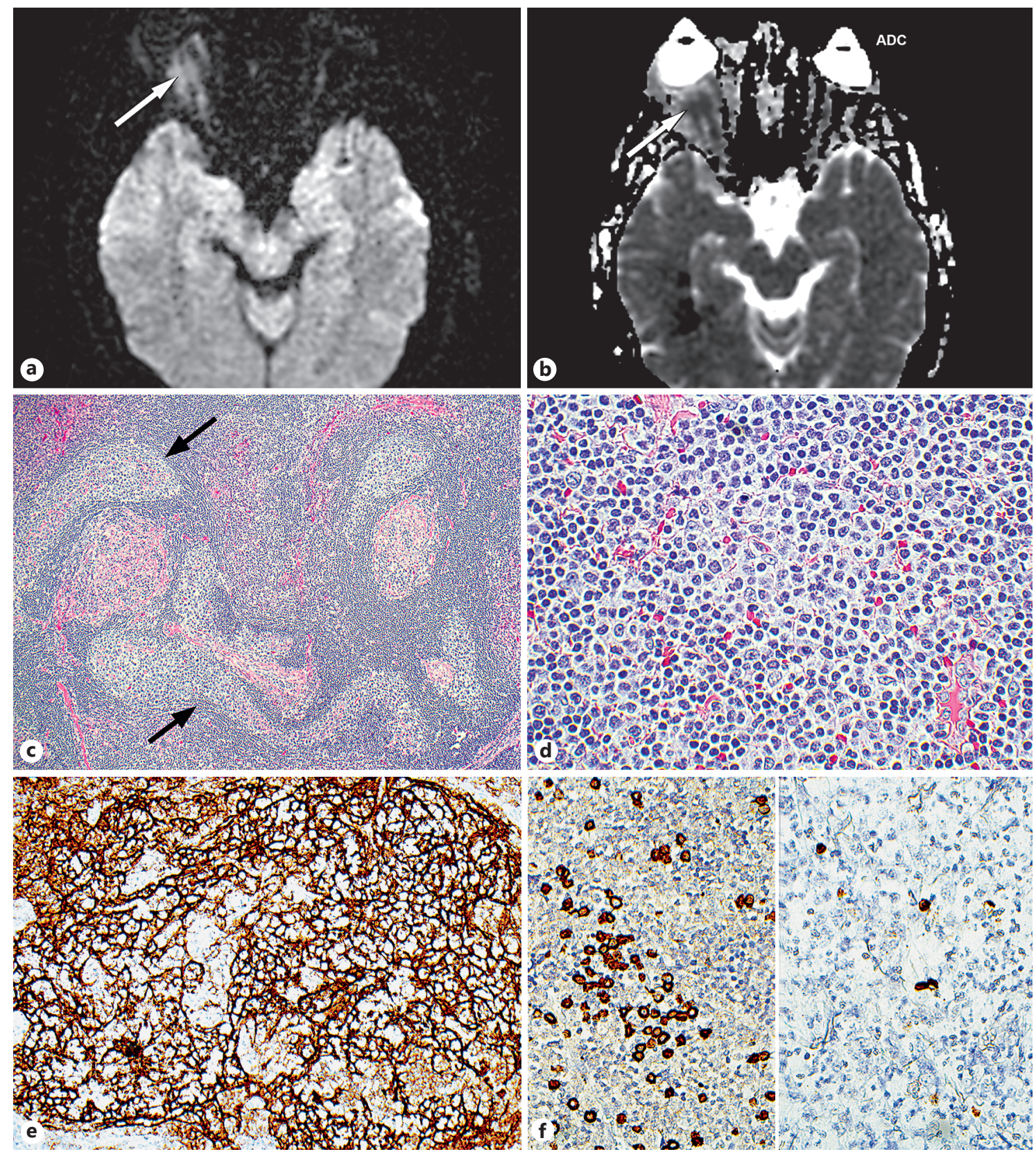

Fig. 3. MRI diffusion restriction and pathologic features. $\mathbf{a}, \mathbf{b}$ The diffusion-weighted image showing a moderately increased signal (arrows) which, coupled with the low signal on the apparent diffusion coefficient (ADC), was considered to represent moderately diminished or intermediate diffusivity, indicating high cellularity. No ADC measurement was available as the study was performed at another institution. c The lesion contained numerous irregularly shaped follicles (arrows) often seen in extranodal marginal zone monoclonal B-cell lymphoma. $\mathbf{d}$ The interfollicular cells were small lymphocytes with slightly irregular nuclei. e The follicles displayed CD21-positive meshworks of dendritic cells that processed antigen for presentation to the follicular cells. $\mathbf{f}$ Left panel: In situ hybridization revealed many kappa-positive B lymphocytes. Right panel: In comparison, scarce lambda-positive cells were identified, establishing that the lesion was monoclonal. c, d Hematoxylin and eosin $(\mathbf{c} \times 10, \mathbf{d} \times 40)$. e, $\mathbf{f}$ Immunoperoxidase reaction, diaminobenzidine chromogen, hematoxylin counterstain $(\mathbf{e} \times 20, \mathbf{f} \times 40)$. 
an early incorrect diagnosis that overlooked valuable diagnostic features seized the stage and was carried forward by a succession of specialists who failed to critically review the flawed basis on which the diagnosis rested. The approach outlined below juxtaposes the diagnostic clinical and radiographic features of both types of lesions to bring out their differences with ultimate histopathologic corroboration. The unusual serpentine growth pattern of the lymphoid tumor, which involved many orbital precincts, was the principal reason for the diagnosticians being led astray.

\section{Clinical Analysis: Lymphoid Tumors}

Orbital lymphoid tumors are the most common orbital mass lesions in adults and are encountered in women slightly more often than men at around 60 years of age [7]. Younger adults can also be afflicted (the present patient was 38 years old), but orbital lymphoid tumors are vanishingly rare in the first two decades of life when a leukemic infiltrate should be suspected [7]. Forty percent of lymphoid tumors are misdiagnosed before biopsy [8]. The onset of proptosis or ocular displacement is usually insidious with minimal discomfort; there may be mild external erythema, motility disturbance, and mild visual decline. The absence of a significant contracting fibrous stroma reduces the potential for more severe symptoms. Fullness of the eyelids does not necessarily indicate tumor involvement, but may rather represent displacement of orbital fat into the eyelids by the tumor. The tumors have a propensity to be located temporally (lacrimal gland) or intraconally.

\section{Clinical Analysis: Vascular Malformations}

In contrast, many vascular malformations are more symptomatic, with prominent associated fibrosis and acute volumetric fluctuations. The most common one is low-flow lymphaticovenous malformation (formerly referred to as a lymphangioma) $[7,9,10]$. These lesions lack a bruit and usually declare themselves in the first decade. They can present as noncircumscribed/infiltrative lesions and feature lymphatic channels of various dimensions, ranging from capillary size to macrocysts, malformed veins, and bundles of smooth muscle and dispersed lymphoid follicles in the interstitium. The associated fibrosis can constrict the optic nerve, causing visual decline and limitation of the motility of the extraocular muscles. Abrupt progression of the proptosis is usually due to intralesional hemorrhage ("chocolate cyst") [7] or subacute inflammation associated with upper respiratory tract infection, which can induce hyperplasia of the interstitial lymphoid aggregates. Nonorbital aids in diagnosis are afforded by the discovery of clear conjunctival "cysts" (actually dilated lymphatic channels), lymphatic vesicles at the eyelid margin, palatal lymphatic vessels, and hygroma coli (cervical masses). More limited and circumscribed lymphaticovenous lesions can be seen in the orbits of adults.

\section{Radiologic Analysis: Lymphoid Tumors}

Computed tomographic scanning, particularly MRI $[11,12]$ with gadolinium contrast injection, is invaluable for defining the soft tissue outlines and perfusion characteristics of lymphoid tumors of the orbit. Bone details are not optimally revealed with MRI, but this is generally not a serious problem because the majority of orbital lymphoid tumors are small-cell lesions of low aggressiveness with little propensity to erode bone. A signal feature is the molding in a putty-like fashion of the lymphoid tissue to the sclera and orbital bones, without bone changes, creating arc-like structures as well as forming sharp demarcation lines denoting fascial boundaries [11]. The lacrimal gland can be diffusely enlarged in an oblong shape, without contiguous bone changes, whereas lacrimal epithelial tumors, in contrast, are unicentric, circumscribed, round or oval, and may create adjacent bone changes. The overall dimensions of the orbit are usually not enlarged. A curious feature in many lymphomatous lesions is the disproportion between the bulkiness of the orbital lesion and the moderateness of the proptosis, implying some degree of lymphoid-induced atrophy or replacement rather than displacement of fat.

In MRI scans, orbital lymphoid lesions generally contact the globe and appear relatively homogeneous. Lateral localization of lymphoid lesions in the orbit leads to lacrimal gland and lateral rectus involvements; multiple thickenings of all of the extraocular muscles can also be seen. These patterns differ from that of thyroid ophthalmopathy, which rarely involves the lateral rectus and does not cause infiltration of the orbital fat. By being located in the orbital fat, lymphoid tumors can wrap around the optic nerve without causing significant visual morbidity, unlike most meningiomas, inflammations, or metastases. The most striking feature of the current patient's lymphoma was its "multifocality," which actually was due to separate large concentrations of lymphocytes interconnected with each other in three dimensions via narrow lymphoid tracts. Lymphomas are isointense to muscle on T1-weighted images, with relatively low signal intensity (appearing as dark areas) on the T2-weighted sequence $[8,12]$. Lesions enhance moderately but homogeneously
86

Ocul Oncol Pathol 2018:4:82-89 DOI: $10.1159 / 00047873$
Jakobiec/Homer/Zakka/Curtin/Fay 
without a prolonged delay since they are directly supplied by the orbital vasculature; they also show diminished diffusion typical of cellular lesions [13].

Diffusion-weighted imaging depends on the reduced diffusivity of water molecules from the elevated backpressure of the orbital tissues in cellular lesions, exampled by lymphomas causing a higher signal. The limitation of water diffusion is also encountered in infarcts and in lesions with barriers provided by viscous extracellular matrices. The term "apparent diffusion coefficient" (Fig. 3b) refers to a quantitative measurement of these phenomena that permits comparisons between individual lesions or different classes of lesions. Fat suppression enhances the clearer visualization of lesions on T1-weighted images after contrast injection. Dynamic contrast-enhanced MRI has been employed to separate benign from malignant lymphoproliferations $[13,14]$. This methodology is based on successive scans linked closely over time in order to trace the speed of perfusion and other characteristics. It is not yet totally clear whether any additional diagnostic malformation can be derived beyond that supplied by studying routine anatomic and enhancement patterns; this technique was not used in the analysis of the current case.

\section{Radiologic Analysis: Vascular Malformations}

Most vascular tumors of the orbit tend to be circumscribed, pseudoencapsulated, unicentric, round lesions (e.g., cavernous hemangiomas, now called venous malformations, and hemangiopericytomas, many of which are now regarded as solitary fibrous tumors when CD34positive). For some lymphaticovenous malformations the term "venolymphatic malformations" can be used when anomalous veins are more prominent than the lymphatic spaces. They are the most common type of infiltrative orbital vascular malformation and are mostly hemodynamically sequestered from the orbital vasculature $[7,9,12]$. Microscopically, they exhibit a diverse range of morphologies constituted by variable mixtures of lymphocytic aggregate, lymphatic channels (including cysts), and veins of different luminal dimensions and mural thicknesses. These features correspondingly create a spectrum of radiologic appearances that encompass delayed and spotty perfusion after gadolinium injection and little evidence of perfusion restriction, which may be evidence in the regions containing hemorrhage or lymphocytic hypercellularity. The volume of the orbit is often enlarged by the congenital presence of vascular malformations, usually without bone erosion. The soft tissue outlines are more irregular than those of lymphoid lesions and generally fail to exhibit the latter's putty-like conformity to surrounding structures [11].

On T1-weighted images, the signal intensity of lymphaticovenous lesions is heterogeneous, mildly hyperintense to muscle, and hypointense to fat. On T2-weighted images, blood-filled cysts may be highlighted with a higher-intensity signal than fat $[7,12]$. Blood products containing paramagnetic methemoglobin are hyperintense on both T1- and T2-weighted sequences, while advanced degradation permitting the accumulation of ferritin and hemosiderin produces low signal intensity on T1- and T2-weighted images. Fluid levels may be present from hemorrhage into cystic spaces. Gadolinium injection discloses retarded lesional perfusion with areas of absent enhancement due to stagnation of the circulation, isolation of the malformed elements, or focal thrombi which may evolve into radiographically detectable phleboliths.

Less common and unrelated vascular malformations with distinctive radiologic findings are congenital arteriovenous high-flow malformations (sometimes called fistulas) of the Wyburn-Mason type [15] and low-flow true venous malformations without bruit [16]. Dynamic contrast-enhanced MRI [17-19] may have something to offer in separating high-flow vascular malformations from low-flow ones and both from lymphoid tumors. On ophthalmoscopy, the retinal vessels accompanying abnormal muscularized orbital vessels are typically tortuous, burnished, and sometimes associated with abnormal fundal vascular patterns and branchings. Doppler studies disclose high flow. The full syndrome includes midbrain arteriovenous malformations together with retinal and orbital lesions. On the other hand, pure venous malformations [16] exhibit only tortuous, patulous veins with some variceal features and lack a bruit. Venous malformations in the head and neck uniformly expand with increasing venous pressure due to the absence of values as can be elicited with head inversion or the Valsalva maneuver. MRI reveals slow or focally absent perfusion conferring overall heterogeneity. There can be conjunctival manifestations along with an orbital venous component, including phlebolith formation indicative of impeded circulation [16].

\section{Pathology and Management}

The biopsy in this case revealed an extranodal marginal zone monoclonal B-cell lymphoma, sometimes also termed mucosa-associated lymphoid tissue lymphoma (MALToma) [7]. This category of lymphoma is composed of small cells with mildly irregular nuclei as well as more discernible cytoplasm and exhibits a variably evi- 
dent follicular architecture which was pronounced in the current case. The follicles were irregularly shaped [20] as opposed to the monotony of those seen in follicular lymphoma (Bcl2-positive follicular B cells). Such irregular follicles are often regarded as reactive (Bcl2-negative follicular B cells), but can be invaded or colonized by the neoplastic population, which is either kappa or lambda light chain monotypic. The interfollicular zones display the neoplastic cells. Once a biopsy-proven diagnosis has been made and a systemic staging workup has failed to detect any nonorbital disease, local radiotherapy in doses of 25-30 Gy is administered [5, 7], with shielding of the globe to achieve maximal protection from radiation. More recently, intralesional anti-CD20 rituximab has been evaluated for efficacy in clinical trials with promising results. The present patient received local radiotherapy and remains tumor-free 11 months after treatment [21].

\section{Summary and Conclusion}

Detailed preoperative analysis of the clinicoradiologic characteristics of an extensive or diffuse orbital mass should allow the distinction between a multicentric lymphoid tumor and an extensive vascular (especially a lymphaticovenous) malformation. Importantly, most diffuse orbital lymphaticovenous lesions are encountered in children, whereas lymphoid tumors characteristically arise in adults - an aspect that should be especially borne in mind by clinicians.

Direct comparison of the MRI characteristics of extensive orbital lymphoid tumors and infiltrative lymphaticovenous malformations reveals major differences in their growth patterns. The former display contoured or molded margins, enhancement with gadolinium, and reduced diffusion because of their hypercellularity. Vascular malformations are heterogeneous, have infiltrative margins, display only spotty perfusion with contrast injection, and do not manifest prominent diffusion restriction, except for regions of hemorrhage or lymphoid hyperplasia.

While not the focus of this paper, which was aimed at correcting the neglected or misinterpreted MRI findings, A- and B-mode ultrasonography can bring additional ancillary information to use in the diagnosis. The uniform composition of lymphoid tumors, which lack a significant fibrous stroma, results in low echographic intralesional reflectivity in A scans and echolucency in B scans. Vascular malformations are septate and possess variably sized blood- or lymph-filled spaces and microcysts, which result in higher reflective acrostic interfaces with marked attenuation of the beam. Ultrasonography has been eclipsed following the advent of MRI scanning with its many refinements, but should still be performed more regularly for orbital masses as an extension of the office examination.

\section{Statement of Ethics}

This study followed the tenets of the Declaration of Helsinki and is compliant with the Health Insurance Portability and Accountability Act's rules and regulations.

\section{Disclosure Statement}

The authors have no conflicts of interest to disclose.

\section{References}

1 Selva D, Rootman J, Crompton J: Orbital lymphoma mimicking optic nerve meningioma. Orbit 2004:23:115-120.

2 Moon WJ, Na DG, Ryoo JW, Kim MJ, Kim YD, Lim DH, Byun HS: Orbital lymphoma and subacute or chronic inflammatory pseudotumor: differentiation with two-phase helical computed tomography. J Comput Assist Tomogr 2003;27:510-516.

3 Moura Neto A, Denardi FC, Delamain MT, Tambascia MA, Vassallo J, Caldato R, ZantutWittmann DE: Orbital lymphoma mimicking ophthalmopathy in a patient with Graves'. Am J Med Sci 2012;344:418-421.

4 Salam A, Saldana M, Zaman N: Orbital cellulitis or lymphoma? A diagnostic challenge. J Laryngol Otol 2005;119:740-742.
5 Eckardt AM, Lemound J, Rana M, Gellrich N: Orbital lymphoma: diagnostic approach and treatment outcome. World J Surg Oncol 2013; 11:73.

6 Galieni P, Polito E, Leccisotti A, Marotta G, Lasi S, Bigazzi C, Bucalossi A, Frezza G, Lauria F: Localized orbital lymphoma. Haematologica 1997;82:436-439.

7 Jakobiec FA, Belyk YR, Font RL: The orbit; in Spencer WH (ed): Ophthalmic Pathology: An Atlas and Textbook. Philadelphia, WB Saunders, 1996, pp 2538-2545, 2686-2736.

8 Gerbino G, Boffano P, Benech R, Baietto F, Gallesio C, Arcuri F, Benech A: Orbital lymphomas: clinical and radiological features. J Craniomaxillofac Surg 2014;42:508-512.
9 Selva D, Strianese D, Bonavolonta G, Rootman J: Orbital venous-lymphatic malformations (lymphangiomas) mimicking cavernous hemangiomas. Am J Ophthalmol 2001;131: 364-370.

10 Nassiri N, Rootman J, Rootman DB, Goldberg RA: Orbital lymphaticovenous malformations: current and future treatments. Surv Ophthalmol 2015;60:383-405.

11 Yeo JH, Jakobiec FA, Abbott GF, Trokel SL Combined clinical and computed tomographic diagnosis of orbital lymphoid tumors. Am J Ophthalmol 1982;94:235-245.

12 Dutton JJ: Radiology of the Orbital Visual Pathways. Philadelphia, Saunders/Elsevier, 2011, pp 276-283. 
13 Sepahdari AR, Politi LS, Aakalu VK, Kim HJ, Razek AA: Diffusion-weighted imaging of orbital masses: multi-institutional data support a 2-ADC threshold model to categorize lesions as benign, malignant, or indeterminate. AJNR Am J Neuroradiol 2014;35:170-175.

$14 \mathrm{Hu} \mathrm{H}, \mathrm{Xu} X Q$, Liu H, Hong XN, Shi HB, Wu FY: Orbital benign and malignant lymphoproliferative disorders: differentiation using semi-quantitative and quantitative analysis of dynamic contrast-enhanced magnetic resonance imaging. Eur J Radiol 2017;88:88-94.

15 Augsburger JJ, Goldberg RE, Shields JA, Mulberger RD, Magargal LE: Changing appearance of retinal arteriovenous malformation. Albrecht Von Graefes Arch Klin Exp Ophthalmol 1980;215:65-70.
16 Jakobiec FA, Werdich XQ, Chodosh J, Freitag SK: An analysis of conjunctival and periocular venous malformations: clinicopathologic and immunohistochemical features with a comparison of racemose and cirsoid lesions. Surv Ophthalmol 2014;59:236-244.

17 O'Connor JP, Tofts PS, Miles KA, Parkes LM, Thompson G, Jackson A: Dynamic contrastenhanced imaging techniques: CT and MRI. Br J Radiol 2011;84(Spec No 2):S112-S120.

18 Turley RS, Lidsky ME, Markovic JN, Shortell CK: Emerging role of contrast-enhanced MRI in diagnosing vascular malformations. Future Cardiol 2014;10:479-486.
19 Tang WQ, Le WJ, Wang PZ, Shi HM, Yu Q Evaluation of soft tissue lesions affecting the masticator space with dynamic contrast enhanced MRI (in Chinese). Zhonghua Kou Qiang Yi Xue Za Zhi 2013;48:711-715.

20 Stacy RC, Jakobiec FA, Schoenfield L, Singh $\mathrm{AD}$ : Unifocal and multifocal reactive lymphoid hyperplasia vs follicular lymphoma of the ocular adnexa. Am J Ophthalmol 2010; 150:412-426.e1. Erratum in: Am J Ophthalmol 2010;159:943.

21 Savino G, Battendieri R, Gari M, Caputo CG, Laurenti L, Blasi MA: Long-term outcomes of primary ocular adnexal lymphoma treatment with intraorbital rituximab injections. J Cancer Res Clin Oncol 2013;139:1251-1255. 